\title{
Comparative microbiota of Rickettsia felis-uninfected and -infected colonized cat fleas, Ctenocephalides felis
}

\author{
Walairat Pornwiroon ${ }^{1}$, Michael T Kearney ${ }^{1}$, Claudia Husseneder ${ }^{2}$, Lane D Foil ${ }^{2}$ and \\ Kevin R Macaluso ${ }^{1}$ \\ ${ }^{1}$ Department of Pathobiological Sciences, School of Veterinary Medicine, Louisiana State University, \\ Baton Rouge, LA, USA and ${ }^{2}$ Department of Entomology, Louisiana State University Agricultural Center, \\ Baton Rouge, LA, USA
}

\begin{abstract}
Fleas serve as arthropod vectors for several emerging and re-emerging infectious disease causing agents including, Rickettsia felis. Although the prevalence of $R$. felis infection in colonies of fleas has been examined, the influence of the $R$. felis infection on flea microbiota has not been investigated. We identified three colonies of cat fleas, Ctenocephalides felis, with varying prevalence of $\boldsymbol{R}$. felis infection (Louisiana State University (LSU), 93.8\%; Professional Laboratory and Research Services Inc. (PLRS), 16.4\%; Elward II (EL), 0\%) and subsequently utilized polymerase chain reaction amplification, restriction fragment length polymorphism analysis and sequencing of the 1.4-kb portions of 16S rRNA genes to examine the diversity of bacteria in the flea populations. A total of 17 different bacterial 16S rRNA gene sequences were identified among the $C$. felis colonies. The prevalence of two Wolbachia species that were identified in each flea colony differed between colonies and $R$. felis-uninfected and -infected fleas. Species richness was unchanged among the $R$. felis-uninfected (LSU, PLRS and EL colonies) and -infected (LSU and PLRS colonies) fleas; however, between $R$. felis-uninfected and -infected fleas within both the LSU and PLRS colonies, $R$. felisuninfected fleas have greater species richness. Diversity indices did not identify a difference in diversity between any of the flea samples. The interaction of endosymbionts within arthropods can widely impact the dissemination of vertically transmitted pathogenic bacteria; and the reciprocal may be true. These results suggest that carriage of $R$. felis has an impact on the richness of flea microbiota.
\end{abstract}

The ISME Journal (2007) 1, 394-402; doi:10.1038/ismej.2007.38; published online 12 July 2007

Subject Category: microbial population and community ecology

Keywords: cat flea; Ctenocephalides felis; endosymbiont; Rickettsia felis

\section{Introduction}

As important human and veterinary parasites, fleas have both a direct physical impact on their hosts and potentially transmit bacterial pathogens. For example, members of the typhus and spotted fever group Rickettsia cause flea-borne rickettsioses with the flea serving as the vector and reservoir. Rickettsia typhi, the etiological agent of murine typhus, is primarily maintained in the rat (Rattus spp) and rat flea (Xenopsylla cheopis) cycle; and a unique opossum/domestic cat and cat flea (Ctenocephalides

Correspondence: Dr KR Macaluso, Department of Pathobiological Sciences, School of Veterinary Medicine, Louisiana State University, Skip Bertman Drive, SVM-3213, Baton Rouge, LA 70803, USA.

E-mail: kmacaluso@vetmed.lsu.edu

Received 7 February 2007; revised and accepted 24 April 2007; published online 12 July 2007 felis) cycle is implicated in transmission of murine typhus in the USA (reviewed in Azad et al., 1997). Spotted fever group Rickettsia are typically associated with ticks; however, there is increasing molecular evidence of one species, Rickettsia felis, in fleas including Ctenocephalides canis (Parola et al., 2003), Pulex irritans (Azad et al., 1997), Anomiopsyllus nudata (Stevenson et al., 2005). Although the list of arthropods infected with $R$. felis continues to grow, $C$. felis remains the primary arthropod host (Adams et al., 1990; Bouyer et al., 2001; La Scola et al., 2002; Pornwiroon et al., 2006). While $R$. felis is maintained in an opossum-flea cycle (Azad et al., 1997; Boostrom et al., 2002), there is also growing evidence that domestic cats also play a role in the maintenance cycle of $R$. felis (Wedincamp and Foil, 2000; Case et al., 2006). The impact of host meal source and/or composition on rickettsial transmission is not known. Another 
ecological consideration for flea-borne rickettsial diseases is the observed experimental coinfection of both $R$. typhi and $R$. felis in the same flea (Noden et al., 1998), although the amount of overlap in naturally infected flea populations remains unclear (Azad et al., 1997; Wiggers et al., 2005).

Microbial diversity has been assessed for wildcaught $C$. felis. Utilizing culture-dependent methods, approximately 53 isolates of Gram-positive and proteobacteria from cat fleas in Australia were isolated on nutrient agar plates (Murrell et al., 2003). Interestingly, there was an ectoparasite (species-specific) restriction for the genera Nocardiopsis, Streptomyces and Flavobacterium to $C$. felis. The isolation and subsequent sequence analysis of bacterial colonies excluded identification of obligate intracellular bacteria such as Wolbachia and Rickettsia. A culture-independent survey for Wolbachia in wild-caught $C$. felis collected in the United States identified Wolbachia in 21\% of fleas assessed as determined by a nested polymerase chain reaction (PCR) and DNA sequencing of the 16S rRNA gene (Gorham et al., 2003). In that study, a number of Wolbachia genotypes were identified with higher prevalence of Wolbachia infection in populations of fleas recovered from domestic animals versus wild animals.

An alternative model for assessing C. felismicrobe interactions utilizes colonized cat fleas. Both the original account (Adams et al., 1990) and subsequent descriptions of $R$. felis (Higgins et al., 1996; Bouyer et al., 2001; La Scola et al., 2002; Pornwiroon et al., 2006) utilized colonized fleas infected with $R$. felis. Likewise, colonized cat fleas are useful for the analysis of transmission events of $R$. felis among flea populations (Wedincamp and Foil, 2000, 2002, 2003). A detailed survey of $R$. felis in eight commercial colonies in the USA provided insight into $R$. felis infection in these populations. The $R$. felis infection rates, as assessed by PCR and restriction fragment length polymorphism analysis (RFLP), varied from $43 \%$ to $93 \%$ in the colonies tested. Interestingly, many of these colonies were either initiated or replenished with fleas from a source that was $R$. felis-infected (Higgins et al., 1994).

The influence of vertically transmitted bacteria (for example, Wolbachia and Spiroplasma) on reproduction has been described for a number of arthropods (reviewed in Bandi et al., 2001), possibly having a broad impact on vertically transmitted rickettsial pathogens. Sex ratio distortion (SRD), favoring female progeny as a continual means for vertical transmission of microorganisms, occurs through a variety of mechanisms including male killing, feminization and parthenogenesis. Additionally, cytoplasmic incompatibility (CI) is an alternative method for reproductive manipulation favoring vertical transmission of microorganisms. SRD has not been identified in fleas; however, either in concert with other microorganisms or alone,
Rickettsia and Wolbachia could potentially manipulate reproductive activity.

Despite the prevalence of $R$. felis and endosymbionts (primarily Wolbachia) in numerous flea colonies and wild-caught fleas, respectively, the interplay between these genera in individual fleas has not been examined. Likewise, previous studies have not accounted for the contribution of the host blood meal source and the nutritional status of the fleas, which has been documented to influence bacterial communities in arthropods (Moreno et al., 2006). As a result of the high level of prevalence and stable vertical transmission of $R$. felis in some colonies of $C$. felis, we hypothesize that $R$. felis directly influences the prevalence of other species of bacteria within the flea. To assess the interplay between endosymbionts and the flea host and the potential impact of infections on vector physiology, we must define the microbial profile before assessing the impact of $R$. felis on the flea host. Toward this objective, we utilized the molecular analysis of the 16S rRNA gene sequence to define the microbiota of three colonies of cat fleas with different blood meal sources and varying levels of $R$. felis infection in the flea population.

\section{Materials and methods}

\section{Source of fleas}

Newly emerged, unfed adult $C$. felis were either obtained from the Louisiana State University (LSU) colony or purchased from commercial vendors. The LSU adult fleas are maintained on domestic shorthair cats at the School of Veterinary Medicine in a manner previously described by Henderson and Foil (1993). A second group of fleas was purchased from Professional Laboratory and Research Services (PLRS) Inc. (Corapeake, NC, USA). This colony is maintained on cats. A third group of fleas was purchased from Elward II (EL) (Soquel, CA, USA). This colony is maintained on sheep blood via an artificial feeding system (Wade and Georgi, 1988).

\section{DNA isolation}

Fleas were washed five times in $70 \%$ ethanol, followed by three washes with DNase-RNase-free water. Fleas were blotted dry and transferred individually to $1.5 \mathrm{ml}$ microcentrifuge tubes containing $20 \mu \mathrm{l}$ of DNase-RNase-free water. Samples were ground with plastic pestles and heated at $95^{\circ} \mathrm{C}$ for $5 \mathrm{~min}$. After brief centrifugation to collect contents, $2.5 \mu \mathrm{l}$ of lysates were used as PCR templates. For comparison of DNA isolation techniques, DNA was isolated from three individual $R$. felis-infected fleas from the LSU colony using the QIAGEN DNeasy tissue kit as described previously (Henry et al., 2007). All DNA samples were utilized as described below for PCR amplification and RFLP analysis and no differences between DNA isolation 
techniques or pooled versus individual flea analyses were identified with respect to total bacterial species present (data not shown).

\section{PCR assessment of rickettsial infection}

Flea lysates from each group were assessed for rickettsial infection using a nested PCR. Two sets of oligonucleotide primers (Integrated DNA Technologies Inc., Coralville, IA, USA) for Rickettsia genusspecific 17-kDa antigen gene, $R r 17.61 \mathrm{p}-R r 17.492 \mathrm{n}$ (Williams et al., 1992) and Rc17.159p-Rc17.308n (5'-GCTTGTTGGAGTAGGTGTAGGTG-3' and $5^{\prime}$ CGCCATTCTACGTTACTACCAC-3'), which generate a $434 \mathrm{bp}$ product and subsequently a $150 \mathrm{bp}$ product, the internal nested region of this gene, were used for PCR amplification. PCR products were amplified using PCR Master Mix (Promega, Madison, WI, USA) with the cycling parameters of an initial denaturation at $94^{\circ} \mathrm{C}$ for $3 \mathrm{~min}$, followed by 35 cycles of denaturation at $94^{\circ} \mathrm{C}$ for $30 \mathrm{~s}$, annealing at $55^{\circ} \mathrm{C}$ for $45 \mathrm{~s}$, extension at $72^{\circ} \mathrm{C}$ for $45 \mathrm{~s}$ and a final extension at $72^{\circ} \mathrm{C}$ for $7 \mathrm{~min}$. Genomic DNA from $R$. montanensis M5/6 (positive control for $17-\mathrm{kDa}$ antigen gene) and water (negative control) were run to ensure proper amplification and lack of contamination, respectively. Amplified products were visualized on ethidium bromide-stained 1.5-2\% agarose gels. For each colony of flea, the $17-\mathrm{kDa}$ antigen gene amplicons were sequenced by the dye terminator method on a 3130 genetic analyzer (Applied Biosystems, Foster City, CA, USA) at School of Veterinary Medicine, LSU.

Amplification and cloning of bacterial 16S rRNA genes The 1.4-kb partial sequence of the 16S rRNA gene was amplified from individual $R$. felis-uninfected or -infected flea lysate using PCR Master Mix (Promega) together with universal bacterial primers fD1 and rP1-3 (Weisburg et al., 1991). The condition used was as follows: $94^{\circ} \mathrm{C}$ for $3 \mathrm{~min}, 35$ cycles of $94^{\circ} \mathrm{C}$ for $30 \mathrm{~s}, 52^{\circ} \mathrm{C}$ for $45 \mathrm{~s}, 72^{\circ} \mathrm{C}$ for $2 \mathrm{~min}$ and $72^{\circ} \mathrm{C}$ for $7 \mathrm{~min}$. $16 \mathrm{~S}$ rRNA gene products were pooled (flea colony/ no. of pools/no. of fleas per pool) for each group of R. felis-uninfected (LSU/1/2; PLRS/2/8; EL/2/8) or -infected fleas (LSU/2/8; PLRS/1/8), gel-purified using Wizard SV Gel Clean-Up System (Promega) and ligated into pCR2.1 vector (Invitrogen, Carlsbad, CA, USA) according to the manufacturer's protocol.

\section{RFLP analysis}

Aliquots of 16S rRNA gene PCR products amplified individually from up to 40 clones from each ligation pool by the fD1 and $\mathrm{rP} 1-3$ primer set were digested with $4 \mathrm{U}$ of restriction enzyme RsaI and AluI (New England Biolabs, Ipswich, MA, USA) at $37^{\circ} \mathrm{C}$ for $3 \mathrm{~h}$ in the manufacturer's recommended reaction buffer. Digests were visualized on ethidium bromide-stained $2.5 \%$ agarose gels.
DNA sequencing and analysis

At least three clones for each RFLP pattern were sequenced as described above. The primers used for sequencing a portion of the 16S rRNA gene were M13F, M13R and the internal primer, 16S rRNAwalk (5'-AGGGTTGCGY[C + T]TCGTTGCK[G + T]GR[A + G]AC-3'). Sequence contigs were assembled using Vector NTI software (Invitrogen) and subjected to BLASTn analysis (http://www.ncbi.nlm.nih.gov/ blast) for similarity to known sequences. All unique sequences of $16 \mathrm{~S}$ rRNA gene were deposited in the GenBank database under accession numbers EF121340 to EF121353.

\section{Molecular phylogenetic analysis}

A multiple sequence alignment for C. felis-associated bacterial 16S rRNA gene sequences, closest database match and most similar sequences for known species was conducted with the sequence alignment for Clustal W. The resulting alignment was used for generation of neighbor-joining and maximum-parsimony gene trees using the Molecular Evolutionary Genetics Analysis (MEGA) version 3.1 software (Kumar et al., 2004). Phylogenetic tree construction utilized Kimura 2-parameter distances. A bootstrap resampling strategy with 500 replicates was employed to assess reliability of the trees.

\section{Statistical analysis}

Differences in microbial communities in fleas were assessed in terms of prevalence of common bacteria (clones) within each colony and between $R$. felisuninfected and -infected cohorts of each colony using Fisher's exact test with a probability less than 0.05 considered a significant difference. Rarefaction was used to compare species richness (number of species) among and between $R$. felis-uninfected and -infected samples. Accumulating curves were generated by selecting 100 random samplings, with replacement, for each sample using EstimateS 7.5 (http:purl.ock.org/estimates), with differences at the $95 \%$ confidence interval level being considered significant. Observed species richness was plotted as a function of the number of clones utilizing the sample-based Mao Tau method (Colwell et al., 2004). The rare-isolate-based Chao1 was used to estimate the total number of bacterial species (Chao, 1984).

Species diversity (number of different species in a cohort of fleas) among the samples was assessed using Fisher's, Shannon's and Simpson's (Simpson and 1 /Simpson) diversity indices.

\section{Results}

PCR assessment of rickettsial infection

To investigate the effect of the carriage of $R$. felis on flea microbiota, rickettsial infection was assessed in three colonies of $C$. felis. A total of 32 (LSU colony), 
55 (PLRS colony) and 50 (EL colony) cat fleas were tested by PCR amplification with the Rr17.61pRr17.492n primer set and $87.5 \%$ (28/32), $0 \%(0 / 55)$ and $0 \%(0 / 50)$, respectively, were found to be positive. The percentage of infection increased to $93.8 \%(30 / 32), 16.4 \%(9 / 55)$ and $0 \%(0 / 50)$ in the corresponding samples using a subsequent nested PCR with the $R c 17.159 \mathrm{p}-R c 17.308 \mathrm{p}$ primer set, indicating high, moderate and no levels of rickettsial infection for LSU, PLRS and EL colonies, respectively. The DNA amplicons of $434 \mathrm{bp}$ (LSU only) and $150 \mathrm{bp}$ (LSU and PLRS) were cloned and sequenced to confirm the specificity of the PCR test; the nucleotide sequences of both amplicons were $100 \%$ matched to sequences reported for $R$. felis in the GenBank database (accession numbers CP000053 and AF195118).

Comparison of bacterial species in C. felis with varying levels of rickettsial infection

The near full-length sequences of the 16S rRNA genes were individually PCR-amplified from randomly selected R. felis-uninfected and -infected $C$. felis samples. Pools of two ( $R$. felis-uninfected LSU fleas only) or eight (all other flea samples) DNA amplicons from flea samples with varying levels of rickettsial infection in each colony were cloned; the 16S rRNA gene PCR products amplified individually from the positive clones were digested by RsaI and AluI. Analysis of 224 clones identified 17 distinct RFLP patterns, indicating that both $R s a \mathrm{I}$ and AluI were needed for accurate differentiation of the clones because the individual enzymes generated a lower number of different operational taxonomic units (Figures 1a and b).

DNA sequences obtained from representatives of each RFLP pattern were compared with known sequences in the GenBank database. RFLP patterns and the corresponding most closely matched bacterial species found in $R$. felis-uninfected and -infected fleas from each colony are shown in Table 1 . A total of 12 bacterial species related to the genera Acidovorax, Acinetobacter, Bergeyella, Bosea, Klebsiella, Methylobacillus, Rickettsia, Spiroplasma, Stenotrophomonas, Staphylococcus and Wolbachia were detected; and, an additional five species were closest matched to various uncultured bacteria. Molecular phylogenetic analysis supports the characterization of $16 \mathrm{~S}$ rRNA gene sequences of C. felis-associated bacteria listed in Table 1 as closely related, sometimes identical, to sequences obtained from BLASTn analysis (Figure 2). Wolbachia pipientis from C. felis $(99 \%$ identity to GenBank accession number AJ628416) and the uncultured ant-associated bacterium (GenBank accession number DQ068830), which groups with Wolbachia under phylogenetic analysis were identified in all of the flea colonies. Significant differences in the prevalence of $W$. pipientis were observed between each colony with the highest prevalence in the EL fleas that were observed to be free of $R$. felis infection by nested PCR with oligonucleotide primers for Rickettsia genus-specific $17-\mathrm{kDa}$ antigen gene. The $R$. felis-uninfected LSU fleas had significantly higher prevalence of Wolbachia

a

RsaI-RFLP pattern

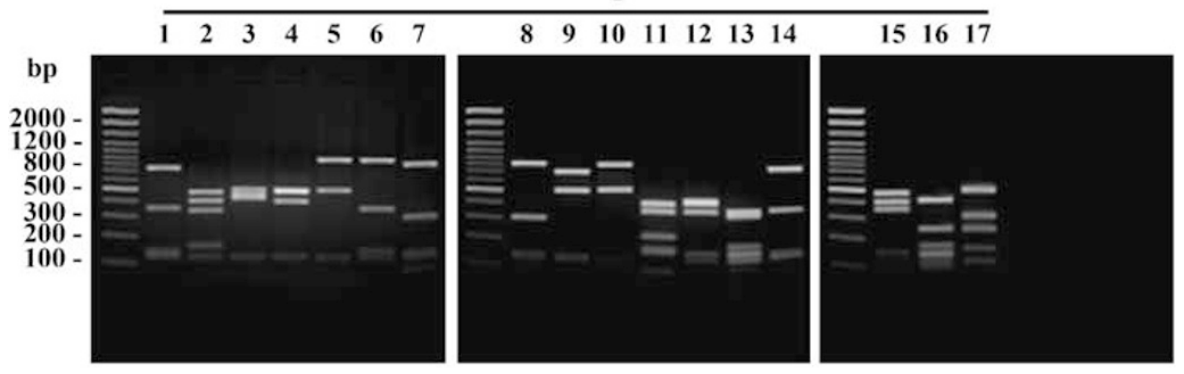

b

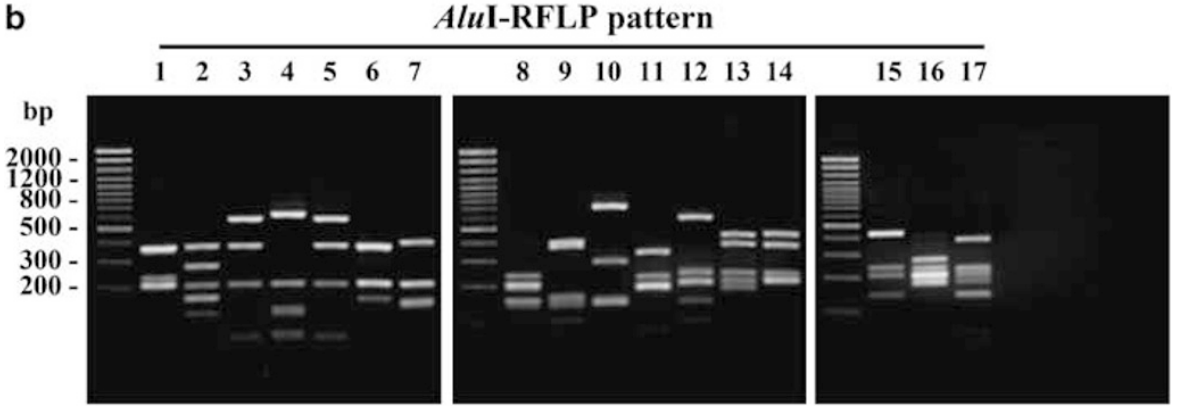

Figure 1 Total 224 positive 16S rRNA clones obtained from Ctenocephalides felis samples with varying levels of Rickettsia felis infection were individually PCR-amplified and the $16 \mathrm{~S}$ rRNA gene PCR products $(\sim 1.4 \mathrm{~kb})$ were digested with RsaI (a), AluI (b). Seventeen distinct restriction fragment length polymorphism (RFLP) patterns were identified. Marker (a 100-bp ladder) sizes are listed to the left of the gel. 
Table 1 Bacteria identified in Rickettsia felis-uninfected and -infected Ctenocephalides felis were differentiated by RFLP analysis and putative identification was assigned based on the closest matched 16S rRNA gene sequence in the GenBank database

\begin{tabular}{|c|c|c|c|c|c|c|c|}
\hline & Flea colony & $\begin{array}{c}\text { RFLP } \\
\text { pattern }^{\mathrm{a}}\end{array}$ & $\begin{array}{l}\text { Length } \\
\quad(b p)\end{array}$ & Closest BLASTn match (accession no.) & $\begin{array}{c}\text { No. } \\
\text { identical/total } \\
\text { (\% identity) }\end{array}$ & $\begin{array}{l}\text { Prevalence } \\
\text { in clones } \\
\text { analyzed } \\
\text { (from no. } \\
\text { of pool) }\end{array}$ & $\begin{array}{c}\text { Accession } \\
\text { no. }{ }^{\mathrm{b}}\end{array}$ \\
\hline \multirow[t]{11}{*}{ PLRS } & \multirow[t]{6}{*}{ Uninfected } & 1 & 1459 & Uncultured bacterium (DQ321554) & 1458/1460 (99) & $9 / 59(2)$ & EF121340 \\
\hline & & 2 & 1467 & Stenotrophomonas sp (DQ256392) & 1466/1467 (99) & $32 / 59(2)$ & EF121341 \\
\hline & & 3 & 1464 & Uncultured bacterium (AF423229) & 1460/1465 (99) & $6 / 59(2)$ & EF121342 \\
\hline & & 4 & 1475 & Staphylococcus saprophyticus (AP008934) & 1475/1475 (100) & $4 / 59(2)$ & - \\
\hline & & 5 & 1462 & Klebsiella pneumoniae (X93214) & 1451/1458 (99) & $3 / 59(2)$ & EF121343 \\
\hline & & 6 & 1460 & Uncultured Acinetobacter sp (DQ366106) & 1458/1460 (99) & $5 / 59(2)$ & EF121344 \\
\hline & \multirow[t]{5}{*}{ R. felis-infected } & 2 & 1467 & Stenotrophomonas sp (DQ256392) & 1466/1467 (99) & $24 / 37$ (1) & - \\
\hline & & 4 & 1475 & Staphylococcus saprophyticus (AP008934) & $1475 / 1475(100)$ & $3 / 37$ (1) & - \\
\hline & & 7 & 1425 & Wolbachia pipientis (AJ628416) & 1387/1391 (99) & $5 / 37(1)$ & EF121345 \\
\hline & & 8 & 1426 & Uncultured bacterium (DQ068830) & 1404/1426 (98) & $2 / 37(1)$ & EF121347 \\
\hline & & 10 & 1444 & Spiroplasma sp (AM087471) & 1437/1444 (99) & $3 / 37(1)$ & EF121346 \\
\hline \multirow[t]{3}{*}{ EL } & \multirow[t]{3}{*}{ Uninfected } & 4 & 1475 & Staphylococcus saprophyticus (AP008934) & 1475/1475 (100) & $3 / 39(2)$ & - \\
\hline & & 7 & 1425 & Wolbachia pipientis (AJ628416) & 1387/1391 (99) & $25 / 39(2)$ & - \\
\hline & & 8 & 1426 & Uncultured bacterium (DQ068830) & 1404/1426 (98) & 11/39 (2) & - \\
\hline \multirow[t]{12}{*}{ LSU } & \multirow[t]{9}{*}{ Uninfected } & 7 & 1425 & Wolbachia pipientis (AJ628416) & 1387/1391 (99) & $3 / 41(1)$ & - \\
\hline & & 8 & 1426 & Uncultured bacterium (DQ068830) & 1404/1426 (98) & $27 / 41(1)$ & - \\
\hline & & 11 & 1439 & Bergeyella sp (AJ575430) & 1425/1429 (99) & 3/41 (1) & EF121348 \\
\hline & & 12 & 1451 & Acidovorax sp (AF458096) & 1449/1451 (99) & $3 / 41(1)$ & EF121349 \\
\hline & & 13 & 1460 & Uncultured bacterium (AF314418) & 1451/1459 (99) & $1 / 41(1)$ & EF121350 \\
\hline & & 14 & 1459 & Acinetobacter junii (AB101444) & $1459 / 1459(100)$ & $1 / 41(1)$ & - \\
\hline & & 15 & 1465 & Methylobacillus flagellatus (СР000284) & 1448/1465 (98) & $1 / 41(1)$ & EF121351 \\
\hline & & 16 & 1453 & Uncultured Burkholderiales bacterium (DQ234135) & 1445/1453 (99) & $1 / 41(1)$ & EF121352 \\
\hline & & 17 & 1408 & Bosea thiooxidans (AF508112) & $1332 / 1406(94)$ & $1 / 41(1)$ & EF121353 \\
\hline & \multirow[t]{3}{*}{ R. felis-infected } & 7 & 1425 & Wolbachia pipientis (AJ628416) & 1387/1391 (99) & $11 / 48(2)$ & - \\
\hline & & 8 & 1426 & Uncultured bacterium (DQ068830) & 1404/1426 (98) & $2 / 48(2)$ & - \\
\hline & & 9 & 1421 & Rickettsia felis URRWXCal2 (CP000053) & 1421/1421 (100) & $35 / 48(2)$ & - \\
\hline
\end{tabular}

Abbreviations: LSU, Louisiana State University; PLRS, Professional Laboratory and Research Services Inc.; EL, Elward II; RFLP, restriction fragment length polymorphism.

${ }^{a}$ RFLP patterns correspond to Figures 1a and b.

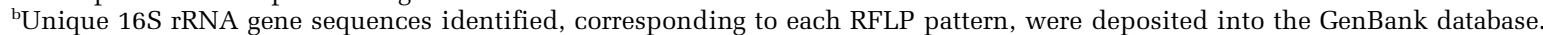

sp (GenBank accession number DQ068830) when compared to other flea samples. Staphylococcus saprophyticus was identified in equal prevalence in clones assessed $(\sim 7 \%)$ from the EL samples and the R. felis-uninfected and -infected PLRS samples. In the PLRS colony, a total of nine unique 16S rRNA gene sequences were identified in two pools of $R$. felis-uninfected and one pool of $R$. felis-infected samples with the predominant $16 \mathrm{~S}$ rRNA clone identified as Stenotrophomonas sp (32/59 clones for $R$. felis-uninfected and 24/37 clones for the R. felisinfected). Other insect-associated bacteria were putatively identified in the PLRS $R$. felis-free samples including Acinetobacter and Klebsiella species. The $R$. felis-infected samples lacked these species, but did have a Spiroplasma sp detected in 3/37 clones assessed. Also, despite the positive amplicon by PCR with Rickettsia genus-specific17$\mathrm{kDa}$ antigen gene primers, the $R$. felis 16S rRNA gene was not detected by this method $(0 / 37)$ in the clones assessed. Combined with the required nested PCR for amplification of the Rickettsia genusspecific $17-\mathrm{kDa}$ antigen gene, these results indicate a low level of $R$. felis infection in these samples.

The predominantly identified clone (35/48) in the $R$. felis-infected samples from the LSU colony was
R. felis. Both Wolbachia spp were identified and constituted the remainder of clones. For the R. felisuninfected LSU sample, DNA amplicons from the two fleas were pooled and nine different clones were identified with the uncultured Wolbachia sp (GenBank accession number DQ068830) as the predominant clone (27/41). Wolbachia pipientis, Acidovorax and Bergeyella were each identified in 3/41 clones assessed. Five of the nine identified sequences closely related to Acinetobacter, Bosea, Methylobacillus and uncultured bacterium were each identified in 1/41 clones assessed.

In most of the samples, the number of clones was enough to capture the species richness; rarefaction demonstrated sample observations leveled off and did not significantly differ from estimated total species richness. With the exception of the $R$. felisuninfected LSU fleas, species richness accumulation curves indicate that estimated species and observed species were comparable for $R$. felisuninfected and -infected fleas from each colony. Species richness did not differ in either $R$. felisuninfected or -infected fleas between the three colonies (LSU, PLRS and EL); therefore, the source of the blood meal did not influence species richness. $R$. felis infection within a colony did influence 


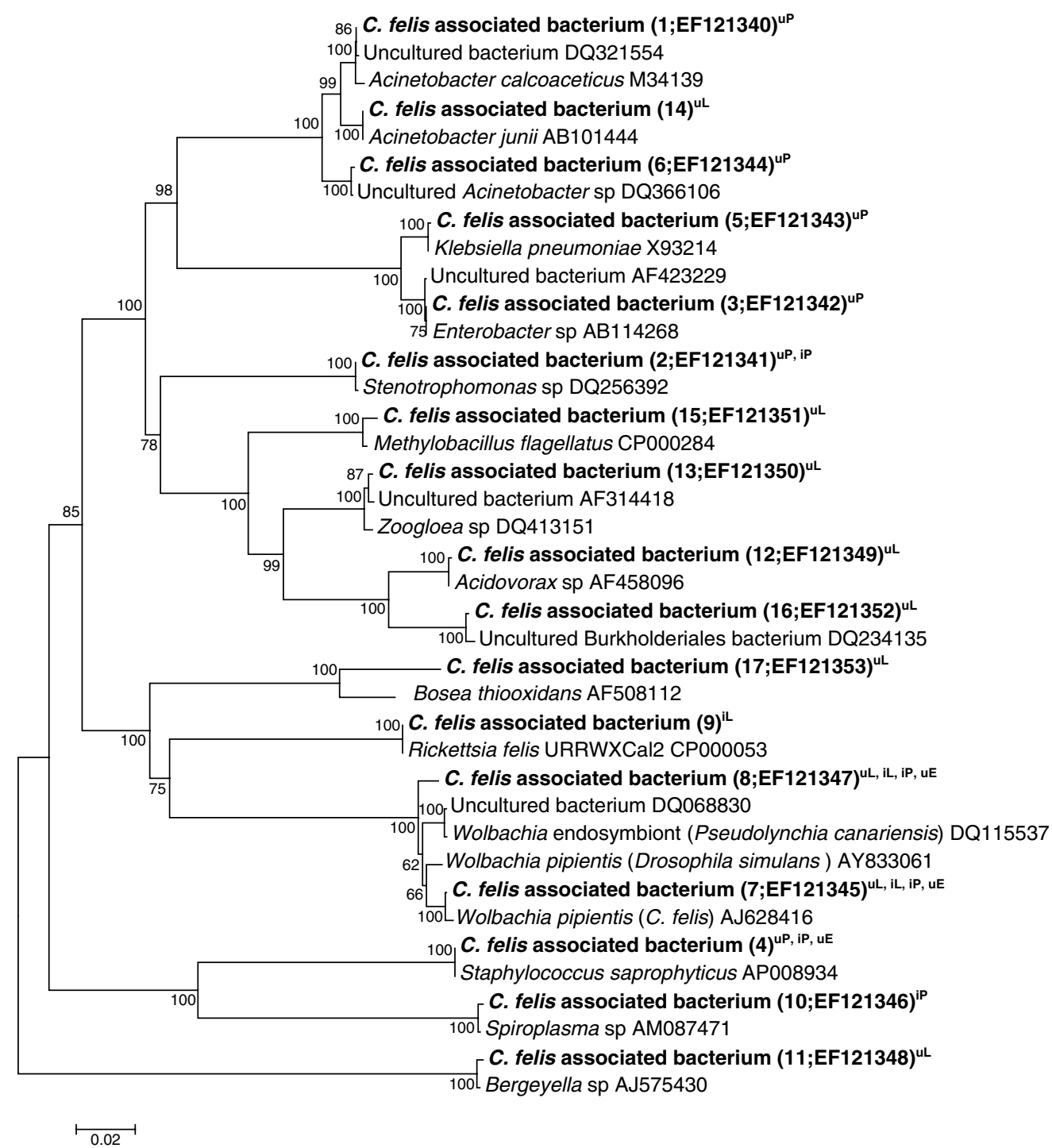

Figure 2 Molecular phylogenetic analysis of Ctenocephalides felis-associated bacteria (RFLP pattern \#; corresponding GenBank accession number) based on 16S rRNA gene sequences identified in Rickettsia felis-uninfected (u) and -infected (i) fleas from the LSU (L), PLRS (P) and EL (E) flea colonies and 16S rRNA gene sequences for the closest match and most similar cultured bacteria. Aligned nucleotide sites were subjected to analysis using the MEGA version 3.1 software. A neighbor-joining tree is shown; maximum parsimony produced a similar topography. The bootstrap values obtained with 500 resamplings are presented in the nodes. LSU, Louisiana State University; PLRS, Professional Laboratory and Research Services Inc.; EL, Elward II; RFLP, restriction fragment length polymorphism.

species richness; decreased richness was observed in $R$. felis-infected fleas when compared to R. felisuninfected fleas within the LSU and PLRS colonies. Although species richness was significantly reduced by the presence of $R$. felis in a colony, the diversity of bacteria present in the colony was not affected, when analyzed with Shannon's and Simpson's diversity indices. The Fisher Alpha index did indicate a greater diversity in $R$. felis-uninfected LSU fleas when compared to $R$. felis-infected fleas.

\section{Discussion}

Despite the recognition of $R$. felis as an emerging pathogen and the cosmopolitan distribution of both the pathogen and $C$. felis, the influence of the $R$. felis infection on the arthropod host has not been examined. The interaction between individual bacterial species within the vector may provide insight into the role of endosymbiont and pathogenic bacteria in flea fitness and define vectorial capacity of the flea population. Our long-term objective is to determine the influence of Rickettsia on the biological parameters of the flea life cycle; therefore, to first define the microbial profile, we surveyed the microbiota of $C$. felis infected with $R$. felis then compared the results to $C$. felis lacking $R$. felis infection.

In the current study, cloned $16 \mathrm{~S}$ rRNA amplicons were subjected to PCR/RFLP, sequencing analysis, and subsequently utilized to compare the diversity 
of bacteria in three colonies of $C$. felis with varying levels of $R$. felis infection in the flea populations. PCR/RFLP is a simple and reproducible method of bacterial differentiation that does not require special equipment and is performed in many laboratories (Shima et al., 2004; Ciantar et al., 2005). Moreover, by using PCR the limitations of traditional culturedependent technique, which requires the isolation and culture of bacteria, is overcome (Ranjard et al., 2000). The widespread use of PCR/RFLP for the characterization of Rickettsia (Regnery et al., 1991; Gage et al., 1994; Roux et al., 1996) including R. felis (Azad et al., 1992; Schriefer et al., 1994; Boostrom et al., 2002) has facilitated rickettsial identification in the absence of culture.

The use of colonized $C$. felis greatly advances research efforts toward the delineation of flea biology, control and pathogen interaction (Thomas et al., 2004). Previous reports identified the presence of $R$. felis (called ELB agent) in eight commercial $C$. felis colonies assessed (Higgins et al., 1994). Three of the colonies assessed by Higgins et al. (1994) were re-examined in the current study. Since the first study was conducted, the prevalence of $R$. felis infection in colonies from California (EL) and North Carolina (PLRS) decreased from $83 \%$ to $0 \%$ and from $50 \%$ to $16.4 \%$, respectively, and increased in the LSU colony from $86 \%$ to $93.8 \%$. Located at different sites, the three colonies (two of which are maintained for commercial purposes) vary in rearing specifications; therefore, it is only possible to speculate what facilitated the changes in prevalence of $R$. felis within the flea populations over time. For example, the LSU colony has been maintained for approximately 3 years without the infusion of outside fleas and within the last 2 years it has experienced a severe population loss followed by a rapid expansion in the population. The high prevalence of $R$. felis in the fleas indicates a relationship between survival and $R$. felis infection.

Murrell et al. (2003) examined the culture-dependent microbial profiles of wild-caught $C$. felis and identified high levels of diversity within the bacterial community, not present in the colonized C. felis. However, there were highly ubiquitous species of bacteria identified in colonized and wild-caught $C$. felis assessed in the current study and previous reports (Gorham et al., 2003; Murrell et al., 2003). S. saprophyticus, which has been described during microbial surveys of fleas, lice and ticks (Murrell et al., 2003), was identified in equal prevalence in the EL samples and the PLRS samples (R. felis-uninfected and -infected). Two species of bacteria, Wolbachia pipientis from C. felis and an uncultured Wolbachia, are present in all three flea colonies. Comparisons of our 16S rRNA gene sequences (Genbank accession numbers EF121345 (1425 bp) and EF121347 (1426 bp)) to the published sequence of Wolbachia endosymbiont from wildcaught $C$. felis (493 bp) (GenBank accession number
AY157503; (Gorham et al., 2003)) exhibited 12 nucleotide differences with varying positions. However, there were 32 nucleotide differences between our two 16S rRNA gene sequences (GenBank accession numbers EF121345 and EF121347).

The contribution of the host blood meal source was compared using a flea colony that is maintained on sheep blood via an artificial feeding system versus a live host (cat) fed fleas. In the current study, we utilized unfed adult fleas to reduce the variability of bacterial infections associated with blood feeding during the adult stage, which has been described for other blood feeding arthropods (Moreno et al., 2006). Neither species richness nor diversity was impacted as a result of colony maintenance on an artificial versus a live host. The lack of significant difference is likely due to the limited differences in larval rearing techniques. However, as with other holometabolous insects, the contribution of the larval diet must be considered (Pidiyar et al., 2004) a source of bacteria in the midgut and, hence, the environmental bacteria identified in this study.

In $R$. felis-infected samples from both the PLRS and LSU colonies, limited richness was associated with rickettsial infection. This is noteworthy because unique insect-associated bacteria were identified in the PLRS $R$. felis-free samples, including Acinetobacter and Klebsiella species. The $R$. felis-infected samples lacked these species, but did have a Spiroplasma sp detected in approximately $8 \%$ of clones. Also, despite the positive amplicon by diagnostic PCR, $R$. felis $16 \mathrm{~S}$ rRNA gene was not detected by this method. The pronounced difference between the prevalence of $R$. felis in flea populations would imply that a pre-existing infection with a vertically transmitted $R$. felis limited microbial richness, but not diversity.

The nutritional status of the arthropod in relation to bacterial composition has been examined for other blood feeding arthropods (Moreno et al., 2006). There was a correlation between life stage, feeding and prevalence of some species of bacteria (for example, Borrelia and Sphingobacteriaceae) in Ixodes scapularis ticks. In the wild-caught flea surveys, most samples were removed from vertebrate hosts, most likely after a blood meal. The microbial profile of the flea in the current study is reflective of the true nature of unfed fleas acquiring bacteria during both transovarial transmission and larval feeding and maintaining them during the molt. Therefore, differences in microflora carried through trans-stadial transmission indicates that $R$. felis influences microbial profiles in immature and adult fleas. Indeed, we observed decreased species richness in $R$. felis-infected fleas in two separate colonies of fleas. Whether $R$. felis infection negatively impacts flea fitness or enhances survival via blocking harmful infections is not examined in this study, but the idea is intriguing. The tissue distribution, if unique for the different species, including 
R. felis, will provide insight into the dynamic microbial interactions within the flea.

The influence of vertically maintained bacteria on reproduction mechanisms of arthropods has been described for a number of bacteria-host associations. Specifically, the contribution of Wolbachia to reproductive manipulation and interspecific interaction between Wolbachia and other symbionts (Goto et al., 2006) is well documented. Parthenogenesis has been reported not to occur in fleas (Zakson-Aiken et al., 1996); however, the contribution of bacteria to reproduction through other mechanisms such as CI has not been examined. The current study has identified the same two Wolbachia species, as determined by $16 \mathrm{~S}$ rRNA gene sequences, in each of the colonies assessed. Remarkably, both Wolbachia were the predominate bacteria in the EL fleas, $64 \%$ and $28 \%$ of clones assessed, respectively, that were observed to be free of $R$. felis infection by diagnostic PCR. Conversely, in the LSU colony with a high prevalence of $R$. felisinfection, these same bacteria (at a ratio of 2:1 in EL) are identified at a ratio of 5:1 with a large decrease in the uncultured Wolbachia (GenBank accession number DQ068830). The ability of Spiroplasma to regulate Wolbachia load and distribution within arthropods has been described (Goto et al., 2006). Whether or not rickettsiae negatively impact other endosymbionts in the fleas has not been determined.

In summary, PCR/RFLP and sequence analysis has been used to characterize microbial profiles and the influence of $R$. felis on these microbial communities of cat fleas. Distinction between PCR-amplified 16S rRNA cloned sequences by RFLP was effective, but required at least two enzymes for definitive separation. The significance of this emerging pathogen, combined with its close association with the arthropod host, make this a worthy model to examine. Specifically, the contribution of $R$. felis to flea fitness and reproductive behavior, and the interacting factors associated with the prevalent Wolbachia infection, should be examined further as this may directly influence rickettsial transmission to vertebrate hosts.

\section{Acknowledgements}

We thank Andre Zanetti for assistance with statistical analysis. This research was supported by the Louisiana Board of Regents (LEQSF), the National Institutes of Health (P20 RR0201595) and the National Institutes of Allergy and Infectious Disease (AI60821).

\section{References}

Adams JR, Schmidtmann ET, Azad AF. (1990). Infection of colonized cat fleas, Ctenocephalides felis (Bouche), with a rickettsia-like microorganism. Am J Trop Med Hyg 43: 400-409.

Azad AF, Radulovic S, Higgins JA, Noden BH, Troyer JM. (1997). Flea-borne rickettsioses: ecologic considerations. Emerg Infect Dis 3: 319-327.
Azad AF, Sacci Jr JB, Nelson WM, Dasch GA, Schmidtman ET, Carl M. (1992). Genetic characterization and transovarial transmission of a novel typhus-like Rickettsia found in cat fleas. Proc Natl Acad Sci USA 89: 43-46.

Bandi C, Dunn AM, Hurst GD, Rigaud T. (2001). Inherited microorganisms, sex-specific virulence and reproductive parasitism. Trends Parasitol 17: 88-94.

Boostrom A, Beier MS, Macaluso JA, Macaluso KR, Sprenger D, Hayes J et al. (2002). Geographic association of Rickettsia felis-infected opossums with human murine typhus, Texas. Emerg Infect Dis 8: 549-554.

Bouyer DH, Stenos J, Crocquet-Valdes P, Moron CG, Popov VL, Zavala-Velazquez JE et al. (2001). Rickettsia felis: molecular characterization of a new member of the spotted fever group. Int J Syst Evol Microbiol 51: 339-347.

Case JB, Chomel B, Nicholson W, Foley JE. (2006). Serological survey of vector-borne zoonotic pathogens in pet cats and cats from animal shelters and feral colonies. J Feline Med Surg 8: 111-117.

Chao A. (1984). Nonparametric-estimation of the number of classes in a population. Scand J Stat 11: 265-270.

Ciantar M, Newman HN, Wilson M, Spratt DA. (2005). Molecular identification of Capnocytophaga spp. via 16S rRNA PCR-restriction fragment length polymorphism analysis. J Clin Microbiol 43: 1894-1901.

Colwell RK, Mao CX, Chang J. (2004). Interpolating, extrapolating, and comparing incidence-based species accumulation curves. Ecology 85: 2717-2727.

Gage KL, Schrumpf ME, Karstens RH, Burgdorfer W, Schwan TG. (1994). DNA typing of rickettsiae in naturally infected ticks using a polymerase chain reaction/restriction fragment length polymorphism system. Am J Trop Med Hyg 50: 247-260.

Gorham CH, Fang QQ, Durden LA. (2003). Wolbachia endosymbionts in fleas (Siphonaptera). J Parasitol 89: 283-289.

Goto S, Anbutsu H, Fukatsu T. (2006). Asymmetrical interactions between Wolbachia and Spiroplasma endosymbionts coexisting in the same insect host. Appl Environ Microbiol 72: 4805-4810.

Henderson G, Foil LD. (1993). Efficacy of diflubenzuron in simulated household and yard conditions against the cat flea Ctenocephalides felis (Bouche) (Siphonoptera: Pulicidae). J Med Entomol 30: 619-621.

Henry KM, Jiang J, Rozmajzl PJ, Azad AF, Macaluso KR, Richards AL. (2007). Development of quantitative real-time PCR assays to detect Rickettsia typhi and Rickettsia felis, the causative agents of murine typhus and flea-borne spotted fever. Mol Cell Probes 21: 17-23.

Higgins JA, Radulovic S, Schriefer ME, Azad AF. (1996). Rickettsia felis: a new species of pathogenic rickettsia isolated from cat fleas. J Clin Microbiol 34: 671-674.

Higgins JA, Sacci Jr JB, Schriefer ME, Endris RG, Azad AF. (1994). Molecular identification of rickettsia-like microorganisms associated with colonized cat fleas (Ctenocephalides felis). Insect Mol Biol 3: 27-33.

Kumar S, Tamura K, Nei M. (2004). MEGA3: integrated software for molecular evolutionary genetics analysis and sequence alignment. Brief Bioinform 5: 150-163.

La Scola B, Meconi S, Fenollar F, Rolain JM, Roux V, Raoult D. (2002). Emended description of Rickettsia felis (Bouyer et al., 2001), a temperature-dependent cultured bacterium. Int J Syst Evol Microbiol 52: 2035-2041. 
Moreno CX, Moy F, Daniels TJ, Godfrey HP, Cabello FC. (2006). Molecular analysis of microbial communities identified in different developmental stages of Ixodes scapularis ticks from Westchester and Dutchess Counties, New York. Environ Microbiol 8: 761-772.

Murrell A, Dobson SJ, Yang X, Lacey E, Barker SC. (2003). A survey of bacterial diversity in ticks, lice and fleas from Australia. Parasitol Res 89: 326-334.

Noden BH, Radulovic S, Higgins JA, Azad AF. (1998). Molecular identification of Rickettsia typhi and $R$. felis in co-infected Ctenocephalides felis (Siphonaptera: Pulicidae). J Med Entomol 35: 410-414.

Parola P, Sanogo OY, Lerdthusnee K, Zeaiter Z, Chauvancy G, Gonzalez JP et al. (2003). Identification of Rickettsia spp. and Bartonella spp. in fleas from the Thai-Myanmar border. Ann NYAcad Sci 990: 173-181.

Pidiyar VJ, Jangid K, Patole MS, Shouche YS. (2004). Studies on cultured and uncultured microbiota of wild Culex quinquefasciatus mosquito midgut based on $16 \mathrm{~S}$ ribosomal RNA gene analysis. Am J Trop Med Hyg 70: 597-603.

Pornwiroon W, Pourciau SS, Foil LD, Macaluso KR. (2006). Rickettsia felis from cat fleas: isolation and culture in a tick-derived cell line. Appl Environ Microbiol 72: 5589-5595.

Ranjard L, Poly F, Nazaret S. (2000). Monitoring complex bacterial communities using culture-independent molecular techniques: application to soil environment. Res Microbiol 151: 167-177.

Regnery RL, Spruill CL, Plikaytis BD. (1991). Genotypic identification of rickettsiae and estimation of intraspecies sequence divergence for portions of two rickettsial genes. J Bacteriol 173: 1576-1589.

Roux V, Fournier P, Raoult D. (1996). Differentiation of spotted fever group rickettsiae by sequencing and analysis of restriction fragment length polymorphism of PCR-amplified DNA of the gene encoding the protein rOmpA. J Clin Microbiol 34: 2058-2065.

Schriefer ME, Sacci Jr JB, Dumler JS, Bullen MG, Azad AF. (1994). Identification of a novel rickettsial infection in a patient diagnosed with murine typhus. J Clin Microbiol 32: 949-954.
Shima K, Terajima J, Sato T, Nishimura K, Tamura K, Watanabe $\mathrm{H}$ et al. (2004). Development of a PCRrestriction fragment length polymorphism assay for the epidemiological analysis of Shiga toxin-producing Escherichia coli. J Clin Microbiol 42: 5205-5213.

Stevenson HL, Labruna MB, Montenieri JA, Kosoy MY, Gage KL, Walker DH. (2005). Detection of Rickettsia felis in a New World flea species, Anomiopsyllus nudata (Siphonaptera: Ctenophthalmidae). J Med Entomol 42: 163-167.

Thomas RE, Macaluso KR, Azad AF. (2004). Laboratory rearing and experimental infection of fleas (Siphonaptera). In: Marquardt WC (ed). The Biology of Disease Vectors. Elsevier: New York, NY, pp 727-732.

Wade SE, Georgi JR. (1988). Survival and reproduction of artificially fed cat fleas, Ctenocephalides felis Bouche (Siphonaptera: Pulicidae). J Med Entomol 25: 186-190.

Wedincamp Jr J, Foil LD. (2000). Infection and seroconversion of cats exposed to cat fleas (Ctenocephalides felis Bouche) infected with Rickettsia felis. J Vector Ecol 25: 123-126.

Wedincamp Jr J, Foil LD. (2002). Vertical transmission of Rickettsia felis in the cat flea (Ctenocephalides felis Bouche). J Vector Ecol 27: 96-101.

Wedincamp Jr J, Foil LD. (2003). Rickettsia felis infection in the cat flea (Siphonaptera: Pulicidae). J Entomol Sci 38: 234-239.

Weisburg WG, Barns SM, Pelletier DA, Lane DJ. (1991). $16 S$ ribosomal DNA amplification for phylogenetic study. J Bacteriol 173: 697-703.

Wiggers RJ, Martin MC, Bouyer D. (2005). Rickettsia felis infection rates in an east Texas population. Tex Med 101: 56-58.

Williams SG, Sacci Jr JB, Schriefer ME, Andersen EM, Fujioka KK, Sorvillo FJ et al. (1992). Typhus and typhuslike rickettsiae associated with opossums and their fleas in Los Angeles County, California. J Clin Microbiol 30: 1758-1762.

Zakson-Aiken M, Gregory LM, Shoop WL. (1996). Reproductive strategies of the cat flea (Siphonaptera: Pulicidae): parthenogenesis and autogeny? J Med Entomol 33: 395-397. 